\title{
Estabilização vertebral segmentar modificada no tratamento de trauma medular em ouriço-cacheiro (Coendou spinosus): relato de caso
}

[Modified segmental vertebral stabilization in the treatment of spinal cord trauma in a Paraguaian hairy dwarf porcupine (Coendou spinosus): case report]

\author{
N.J. Tagliari ${ }^{1}$, B.Z. Silva ${ }^{1}$, E.A.R. Santos ${ }^{1}$, M.E. Baier ${ }^{1}$, P.C.S. Gonzalez ${ }^{2}$, M.M. Alievi ${ }^{2 *}$ \\ ${ }^{1}$ Aluno de pós-graduação - Universidade Federal do Rio Grande do Sul - Porto Alegre, RS \\ ${ }^{2}$ Universidade Federal do Rio Grande do Sul - Porto Alegre, RS
}

\begin{abstract}
RESUMO
Uma fêmea, adulta, de ouriço-cacheiro (Coendou spinosus, F. Cuvier, 1823), foi atendida com histórico de trauma medular. No exame neurológico, foi constatada paraplegia com nocicepção preservada e sinais compatíveis com choque espinhal. Exame radiográfico e mielografia apontaram presença de fratura e luxação vertebral torácica entre T10-11. O paciente foi submetido à técnica de estabilização vertebral segmentar modificada, com pinos de Steinmann e fios de cerclagem. A técnica utilizada mostrou-se eficaz na estabilização da fratura/luxação vertebral e na recuperação dos sinais neurológicos. O paciente apresentou recuperação progressiva da deambulação. Para o conhecimento dos autores, este é o primeiro relato utilizando estabilização vertebral segmentar modificada para o tratamento de fratura/luxação vertebral torácica em Coendou spinosus.
\end{abstract}

Palavras-chave: estabilização segmentar modificada, trauma medular, ouriço-cacheiro

\begin{abstract}
An female, adult Paraguayan hairy dwarf porcupine (Coendou spinosus, F. Cuvier, 1823) was referred to the Veterinary Hospital with clinical history of spinal cord injury. In the neurological examination, paraplegia with preserved nociception compatible with signs of spinal shock were observed. Radiographic and myelographic examinations showed evidence of a vertebral dislocation and fracture between the 10th and 11th thoracic vertebrae. The patient underwent surgical stabilization of the lesion using the modified segmental vertebral stabilization technique with Steinmann pins and cerclage wires. Under the conditions reported in this case, the technique was effective in stabilizing vertebral fracture/dislocation and in recovering neurological functions. The patient presented progressive recovery from ambulation. To the authors' knowledge, this is the first case report that performed modified segment stabilization for thoracic vertebral fracture/luxation treatment in Coendou spinosus.
\end{abstract}

Keywords: modified segmental stabilization, spinal cord trauma, paraguaian hairy dwarf porcupine

\section{INTRODUÇÃO}

O ouriço-cacheiro (Coendou spinosus) é uma espécie de roedor encontrada no sudeste brasileiro, leste paraguaio, norte uruguaio e nordeste argentino, o qual ocupa diversos habitats, incluindo florestas secundárias e nas proximidades de comunidades humanas (Roach e Naylor, 2016). A ação antrópica no meio

Recebido em 27 de agosto de 2017

Aceito em 16 de dezembro de 2017

*Autor para correspondência (corresponding author)

E-mail: marcelo.alievi@ufrgs.br ambiente, com consequente migração de espécies para o espaço urbano, favorece o aumento de traumatismos na fauna silvestre (Bastos et al., 2003).

Fraturas e luxações vertebrais são consideradas as afecções mais comuns entre as emergências neurológicas e apresentam elevado risco de danos medulares permanentes, o que demanda uma abordagem clínico-cirúrgica rápida e precisa 
para limitar complicações e favorecer o prognóstico (Weh e Kraus, 2012). Traumas vertebrais por projéteis de armas de fogo, interação animal e atropelamento podem levar a sequelas, como perda parcial ou completa das funções motoras, sensoriais e viscerais (Bruce et al., 2008). O segmento vertebral toracolombar é citado como o de maior acometimento por lesões traumáticas em cães e gatos (Bruce et al., 2008).

O correto diagnóstico necessita da associação entre anamnese, sinais clínicos agudos, exame neurológico e diagnóstico por imagem da coluna vertebral e medula espinhal (Voss e Montavon, 2004). Durante exame físico ou manejo do paciente traumatizado, recomenda-se muito cuidado na manipulação, para evitar lesão espinhal iatrogênica provocada por movimentos desnecessários ou incorretos. O presente relato descreve a técnica de estabilização vertebral segmentar modificada para o tratamento de fratura/luxação vertebral torácica em Coendou spinosus, destacando-se a relevância do tratamento em tempo hábil na presença de dor profunda, para a recuperação das funções neurológicos e a retomada da deambulação.

\section{CASUÍSTICA}

Uma fêmea, adulta, de ouriço-cacheiro (Coendou spinosus), pesando $1,6 \mathrm{~kg}$, vítima de mordedura por cães, foi encaminhada ao Núcleo de Conservação e Reabilitação de Animais Silvestres (Preservas) do Hospital de Clínicas Veterinárias da Universidade Federal do Rio Grande do Sul (UFRGS). Realizou-se exame físico, no qual foram constatados, na região da $10^{\mathrm{a}}$ e $11^{\mathrm{a}}$ vértebras torácicas, laceração de pele de 1 centímetro de comprimento, desvio da coluna vertebral (escoliose) para a direita e aumento de volume associado à hiperestesia nesta região. Ao exame neurológico, os pares de nervos cranianos apresentaram resposta adequada, e os membros torácicos encontravam-se com reações posturais normais e reflexos espinhais preservados. Nos membros pélvicos, observaram-se ausência de propriocepção, paraplegia flácida, nocicepção preservada e redução do tônus muscular, reflexo flexor e reflexo patelar. Os parâmetros hematológicos e bioquímicos estavam dentro da normalidade para a espécie.

Para realização do exame radiográfico, foi executada contenção química com midazolam (0,5mg/kg, IM) e meperidina (10mg/kg, IM). Inicialmente, foi realizado exame radiográfico simples, que demonstrou fratura e luxação vertebral (FLV) entre a $10^{\mathrm{a}}$ e $11^{\mathrm{a}}$ vértebras torácicas e fratura de múltiplas costelas (Fig. 1). Foi realizada bandagem de proteção do ferimento e da parede torácica e instituída analgesia com tramadol (10mg/kg, SC, TID) e dipirona (20mg/kg, IV, TID). Para antibioticoterapia, foi utilizado enrofloxacina (10mg/kg, IV, BID).

Após 12 horas do exame radiográfico simples, foi realizada mielografia. A medicação préanestésica foi a mesma citada anteriormente no exame radiográfico, porém a indução anestésica e a manutenção da anestesia foram feitas com auxílio de máscara facial com isoflurano vaporizado em $100 \%$ de oxigênio. A mielografia foi realizada com agulha espinhal 25G pelo acesso entre a quinta e sexta vértebras lombares, na dose estimada por alometria de $0,5 \mathrm{~mL} / \mathrm{kg}$ de iohexol. As imagens revelaram desvio dorsal e adelgaçamento da coluna de contraste ventral e dorsal entre T10-11 além de redução do preenchimento do contraste, evidenciado pela menor radiopacidade do canal medular entre T10-11, e integridade da dura-máter (Fig. 1).

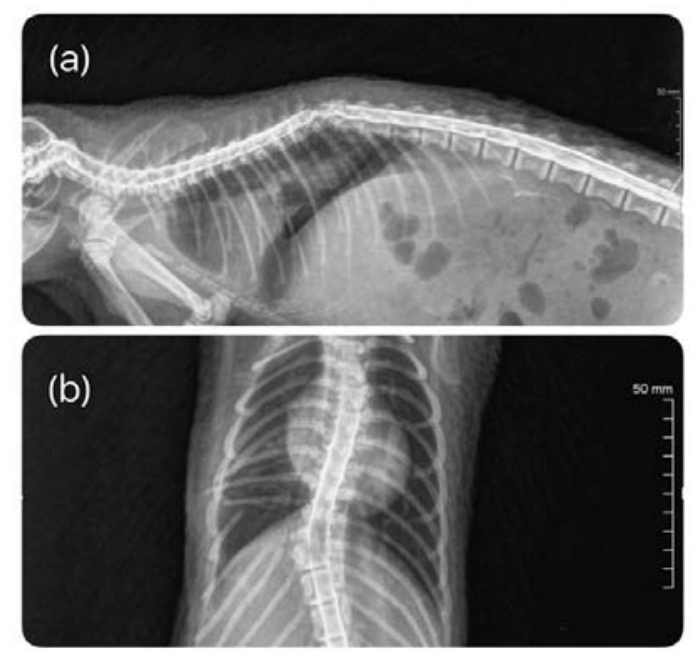

Figura 1. Mielografia em Coendou spinosus. Projeção laterolateral (a) e ventrodorsal (b) demonstrando fratura e luxação vertebral entre a $10^{\mathrm{a}}$ e $11^{\mathrm{a}}$ vértebras torácicas, fratura de múltiplas costelas, desvio dorsal e adelgaçamento da coluna de contraste ventral e dorsal entre T10-11, além de redução do preenchimento do contraste, evidenciado pela menor radiopacidade do canal medular entre T10-11, e integridade da duramáter. 
Com base nos sinais clínicos, nos achados radiográficos e mielográficos, optou-se pela correção cirúrgica utilizando-se a técnica de estabilização vertebral segmentar modificada, sendo realizada 24 horas após a mielografia. O protocolo de MPA incluiu metadona $(0,3 \mathrm{mg} / \mathrm{kg}$, IM) e midazolam $(0,5 \mathrm{mg} / \mathrm{kg}$, IM). A indução anestésica foi realizada com auxílio de máscara facial com isoflurano vaporizado ao efeito em $100 \%$ de oxigênio. A anestesia geral foi mantida com isoflurano vaporizado ao efeito em $100 \%$ de oxigênio mediante uma traqueostomia temporária com tubo endotraqueal 2,0 sem balonete. Parâmetros anestésicos, como oximetria de pulso, eletrocardiografia, temperatura esofágica e pressão arterial não invasiva, foram aferidos por meio de um monitor multiparamétrico, não sendo observadas intercorrências durante o procedimento.

Após o posicionamento do paciente em decúbito esternal e a antissepsia com gluconato de clorexidina $4 \%$, procedeu-se à incisão de pele, tendo como referência três processos espinhosos craniais e três caudais à fratura/luxação vertebral. Ao lado direito da lesão, foi observada ruptura completa dos grupos musculares epaxiais torácicos, criando-se uma comunicação com a cavidade torácica, que possibilitou a visualização do parênquima pulmonar. A cavidade torácica foi lavada com solução de cloreto de sódio $0,9 \%$ $(500 \mathrm{~mL} / \mathrm{kg})$ e a musculatura lacerada foi suturada com mononáilon 3-0 em padrão interrompido simples, o que permitiu continuidade ao procedimento de estabilização vertebral. A musculatura epaxial foi rebatida lateralmente até expor três processos espinhosos e facetas articulares craniais e caudais à lesão. A luxação foi reduzida com auxílio de pinças de apreensão ósseas ancoradas aos processos espinhosos dorsais. Com a utilização de introdutor manual, foram confeccionados orifícios através da base dos processos espinhosos, e fios de aço foram passados através deles. Na sequência, foram introduzidos os pinos de Steinmann no terceiro espaço intervertebral cranial e caudal à lesão. Os pinos foram acomodados em "U" entre os processos espinhosos e as facetas articulares das vértebras. Os fios de aço pré-colocados foram retorcidos ao redor dos pinos de Steinmann (Fig. 2).

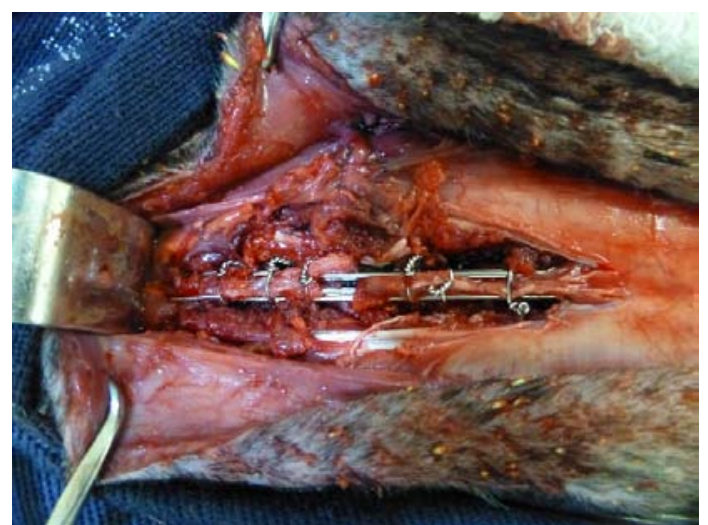

Figura 2. Reparação de fratura e luxação vertebral em Coendou spinosus. Técnica de estabilização vertebral segmentar modificada. Transfixação dos processos espinhosos com fios de aço retorcidos ao redor dos pinos de Steinmann, demostrando redução e alinhamento do canal vertebral.

O local da cirurgia foi lavado com solução de cloreto de sódio 0,9\%, e a musculatura epaxial foi reduzida com mononáilon 3-0, padrão contínuo, seguido de redução do tecido subcutâneo com o mesmo fio e o mesmo padrão de sutura. A dermorrafia foi realizada com mononáilon 3-0, padrão interrompido simples. Para o procedimento de traqueorrafia, foi utilizado o mesmo fio e padrão de sutura. A recuperação cirúrgico-anestésica transcorreu sem intercorrências. Realizou-se exame radiográfico pós-cirúrgico imediato para controle e avaliação (Fig. 3)

O pós-operatório imediato constou de dexametasona $(0,5 \mathrm{mg} / \mathrm{kg}$, IV, dose única) e metadona $(0,3 \mathrm{mg} / \mathrm{kg}$ SC, TID, um dia). O tratamento clínico abrangeu enrofloxacina (10mg/kg, IV, BID, 21 dias), metronidazol (20mg/kg, IV, BID, 10 dias), meloxicam $(0,5 \mathrm{mg} / \mathrm{kg}$, IM, SID, sete dias), tramadol (10mg/kg, SC, TID, 10 dias), dipirona (20mg/kg, IV, TID, 15 dias), ranitidina (2mg/kg, VO, SID, 15 dias) e simeticona (70mg/kg, VO, TID, 20 dias). Ainda foi introduzida suplementação nutricional com glicolpet ${ }^{\circledR}(0,5 \mathrm{~mL} / \mathrm{kg} \mathrm{VO}$, SID, 40 dias) e probiótico (0,5g/kg VO, SID, 60 dias). Repouso em gaiola durante 30 dias e compressão vesical três vezes ao dia foram instituídos como complementares ao tratamento clínico. 

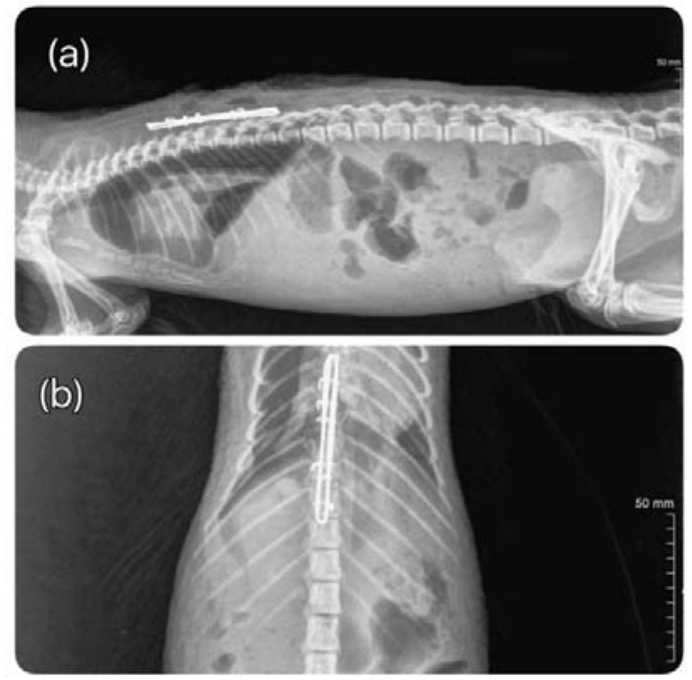

Figura 3. Exame radiográfico pós-cirúrgico imediato em Coendou spinosus. Projeção laterolateral (a) e ventrodorsal (b), demonstrando implante metálico dorsal às vértebras torácicas e correto alinhamento da coluna vertebral. Elevação da silhueta cardíaca em relação ao esterno, aumento da densidade do pulmão atelectásico e hiperluscência do campo pulmonar, compatível com pneumotórax.

O animal apresentou pneumotórax transitório nas primeiras quatro horas pós-cirúrgicas com drenagem de $20 \mathrm{~mL}$ de ar do hemitórax direito. Houve adequada cicatrização da ferida cirúrgica e as suturas foram removidas 10 dias após a cirurgia.

A propriocepção retornou gradativamente a partir do $10^{\circ}$ dia de pós-operatório, e, no $21^{\circ}$ dia, o animal apresentava paraparesia ambulatória. A micção normalizou gradativamente, e, consequentemente, a frequência das compressões vesicais foram reduzidas. No $15^{\circ}$ dia de póscirúrgico, o animal estava continente. Nova radiografia simples foi realizada com 30 dias de pós-cirúrgico, demonstrando moderado desvio lateral à direita (escoliose) e discreto desvio dorsal (cifose) do segmento torácico da coluna vertebral, sendo o núcleo de desvio observado entre a $10^{\mathrm{a}}$ e a $11^{\mathrm{a}}$ vértebra torácica. Entretanto, o animal não apresentou qualquer prejuízo na recuperação e evolução clínica.

Passados três meses da cirurgia, o animal apresentava notável evolução clínica e deambulação normal. Com seis meses de pósoperatório, foi realizado exame radiográfico controle, e o quadro clínico seguiu estável. Em conjunto com o órgão ambiental competente, foi definido que o animal será mantido em cativeiro, dado o tempo de internação e a complexidade de sua lesão e tratamento cirúrgico.

\section{DISCUSSÃO}

Acidentes envolvendo ouriço-cacheiro em ambientes urbanos podem ser observados em diversas cidades brasileiras, onde a sua presença é comum e percebida pelo alto número de acidentes envolvendo cães reportados em atendimentos veterinários (Lange e Schmidt, 2014).

Conforme Sweet e Waters (1991), como as feridas penetrantes sempre devem ser consideradas contaminadas, os autores indicam a aplicação de bandagem para proteção do ferimento e da parede torácica e antibioticoterapia de amplo espectro, corroborando a conduta adotada no primeiro momento do atendimento. Anamnese, sinais clínicos agudos, exame neurológico e exames complementares da coluna vertebral (Voss e Montavon, 2004) auxiliaram o correto diagnóstico e permitiram um adequado planejamento cirúrgico. O exame radiográfico é rápido e de baixo custo para avaliação inicial do paciente, embora tenha suas limitações por possuir baixo valor preditivo e moderada sensibilidade na determinação da presença de fragmentos ósseos no interior do canal vertebral e de discretas subluxações (Kinns et al., 2006).

O anel do disco intervertebral e as facetas articulares apresentam um papel importante na estabilidade vertebral; assim, luxações vertebrais devem ser consideradas lesões potencialmente instáveis, sobretudo quando houver fratura de facetas articulares (Shires et al., 1991). Portanto, a instabilidade comprovada pela luxação, juntamente com a mielografia, que apontou desvio dorsal, adelgaçamento da coluna de contraste ventral e dorsal entre T10-11 e integridade da dura-máter, além de nocicepção preservada, confirmou a necessidade de alinhamento e estabilização cirúrgica do segmento vertebral. Este é o tratamento de escolha para animais com déficits graves, lesões instáveis, fraturas deslocadas e evidências de compressão medular (Olby, 2010). Placas, pinos, polimetilmetacrilato e estabilização segmentar 
modificada são os métodos de estabilização mais utilizados na região toracolombar de cães (Mcanulty et al., 1986).

Lesões no segmento torácico caracterizam sinais do tipo neurônio motor superior (NMS), porém uma exceção à regra no contexto do paciente emergencial é que lesões transversas, hiperagudas e severas da coluna espinhal toracolombar, normalmente, demonstram hipotonia dos membros pélvicos e reflexos espinhais reduzidos durante alguns dias após o decorrido (Olby, 2010). No presente relato, o animal apresentava lesão no segmento torácico e sinais de neurônio motor inferior (NMI) nos membros pélvicos, como tônus muscular, reflexo flexor e reflexo patelar reduzidos. Isso é comparado à medicina humana com uma condição similar chamada choque espinhal. A razão pela qual a interrupção da via do NMS causa sinais de NMI é pobremente entendida (Olby, 2010).

Justifica-se o uso da técnica de estabilização vertebral segmentar modificada no presente caso por se tratar de uma técnica de baixo custo e de fácil aplicação na região toracolombar, além de exigir discreta dissecção muscular e poder ser utilizada em animais de portes variados (Mcanulty et al., 1986). Os objetivos foram alcançados, visto que houve o alinhando do canal vertebral e a estabilização simétrica da coluna vertebral, além do que foram preservados os ligamentos supraespinhosos e interespinhosos, o que ajuda a preservar a estabilidade do segmento (Bruecker, 1996). Voss e Montavon (2004) relataram, em cães e gatos, recuperação neurológica satisfatória ou completa, quando utilizaram essa técnica em 79\% dos pacientes.

A nocicepção preservada nos membros pélvicos e a ausência de secção medular comprovada pelo adelgaçamento da coluna de contraste ventral e dorsal entre T10 e T11 são fatores prognósticos favoráveis, conforme Weh e Kraus (2012), e, associadas à adequada estabilização vertebral segmentar modificada, contribuíram para a recuperação e o prognóstico neurológico favorável.

Não foram observadas complicações graves, como migração dos pinos ou fratura por fadiga dos pinos e fios de aço. O moderado desvio dorsolateral do foco da luxação verificado radiograficamente aos 30 dias pode ser atribuído à dificuldade de ancoragem dos fios de aço nos processos articulares em virtude do seu tamanho reduzido. Esse tipo de ancoragem, seja nas facetas articulares, no osso cortical da lâmina dorsal, seja na base dos processos espinhosos dorsais, proporciona pontos fortes de apoio e diminui o potencial para "pullout" das cerclagens e fratura óssea, que levam à perda da estabilização (Mcanulty et al., 1986).

\section{CONCLUSÃO}

Conclui-se que a utilização de estabilização vertebral segmentar modificada para correção de fratura/luxação vertebral em ouriço-cacheiro (Coendou spinosus), tratado em tempo hábil, pode assegurar adequada estabilização e retorno das funções neurológicas. Tomografia computadorizada e/ou ressonância magnética podem ser necessárias para melhor avaliação da medula espinhal e da coluna vertebral em três dimensões, podendo, inclusive, auxiliar no prognóstico, na tomada de decisão e no melhor planejamento cirúrgico.

\section{REFERÊNCIAS}

BASTOS, R.P.; MOTTA, J.A.O.; LIMA, L.P. et al. Anfíbios da floresta nacional de Silvânia. Goiânia: Semarh, 2003. 82p.

BRUCE, C.W.; BRISSON, B.A.; GYSELINCK K. Spinal fracture and luxation in dogs and cats: a retrospective evaluation of 95 cases. Vet. Comp. Orthop. Traumatol., v.21, p.280-284, 2008.

BRUECKER, K.A. Principles of vertebral fracture management. Semin. Ciênc. Agrár., v.11, p.259-272, 1996.

KINNS, J.; MAI, W.; SEILER, G. et al. Radiographic sensitivity and negative predictive value for acute canine spinal trauma. Vet. Radiol. Ultrasound, v.47, p.563-570, 2006.

LANGE, R.R.; SCHMIDT, E.M.S. Rodentia roedores selvagens (capivara, cutia, paca e ouriço). In: CUBAS Z.S.; SILVA J.C.R.; CATÃO-DIAS, J.L. (Eds.). Tratado de animais selvagens. São Paulo: Roca, 2014. p.1137-1168.

MCANULTY, J.F.; LENEHAN, T.M.; MALETZ, L.M. Modified segmental spinal stabilization in repair of spinal fractures and luxations in dogs. Vet Surg., v.15, p.143-149, 1986. 
OLBY N. The pathogenesis and treatment of acute spinal cord injuries in dogs. Clin. N. Am. Small Anim. Pract., v.40, p.791-807, 2010.

ROACH, N.; NAYLOR, L. Coendou spinosus. $2016 . \quad$ Available in: <http://dx.doi.org/10.2305/IUCN.UK.20162.RLTS.T20630A22213974.en>. Accessed in: 22 Jun. 2017.

SHIRES, P.K.; WALDRON, D.R.; HEDLUND, C.S. et al. A biomechanical study of rotational instability in unaltered and surgically altered canine thoracolumbar vertebral motion units. Prog. Vet. Neurol., v.6, p.6-14, 1991.
SWEET, D.C.; WATERS, D.J. The role of surgery in the management of dogs with pathologic conditions of the thorax: part II. Comp. Cont. Educ. Pract., v.13, p.1771-1776, 1991.

VOSS, K.; MONTAVON, P.M. Tension band stabilization of fractures and luxations of the thoracolumbar vertebrae in dogs and cats: 38 cases (1993-2002). J. Am. Vet. Med. Assoc., v.1, p.78-83, 2004.

WEH, M.; KRAUS, K.H. Spinal fractures and luxations. In: TOBIAS K.M.; JOHNSTON S.A. Veterinary surgery: small animal. Philadelphia: Saunders Elsevier, 2012. p.487-503. 